\title{
Research on the Organization Performance of AgSnO2 Electrical Contact Materials
}

\author{
Xingrong JIANG ${ }^{1, a}$ \\ ${ }^{1}$ sichuan technology and business college, Dujiangyan 611837,China \\ ajiangxingrong@126.com
}

Keywords: AgSno2; Organization Performance; Electrical Contact Materials

\begin{abstract}
Metal oxide electrical contact materials because it has excellent corrosion resistance, good weldability, high conductivity and high thermal conductivity, in the general application of relay is more and more common. In this paper, the research of system adopts the technology of the preparation of AgSnO2 materials microstructure of structure and the influence of additives on the microstructure and physical and mechanical properties of materials and electrical contact performance, corrosion resistance, and comparison with the conventional powder metallurgy method of AgSnO2 on microstructure and performance difference, the difference between a new preparation technology for the development of $\mathrm{AgSnO} 2$ materials and provide theoretical basis and practical application.
\end{abstract}

\section{Introduction}

In electrical contact materials, silver metal oxide (AgMeO) materials because has good resistance to abrasion, fusion welding resistance and electrical conductivity, is widely used in low-voltage electrical appliances. And silver cadmium oxide (AgCdO) because it has excellent electric arc erosion resistance, fusion welding resistance so was widely used in from a few volts to kv in a variety of low-voltage electrical appliances. The material because of a series of excellent performance has been called the universal contact [1]. But because AgCdO materials in use process inevitably produce tin poison, due to the urgent requirement of environmental protection, hoping to develop a kind of material to replace AgCdO, after years of effort, people developed AgZnO, AgSnO2, AgNiO, and other series of silver metal oxide contact materials. Studies have shown that AgSnO2 materials due to exhibit more excellent fusion welding resistance and electric resistance loss performance, is the most promising to replace the silver oxide of new non-toxic materials [2], but as a result of material preparation technology, processing technology and equipment, silver metal oxide material especially the AgSnO2 is not at home to realize large-scale production, must carry on the deep research, as early as possible in order to make the AgSnO2 materials for practical applications, this topic is proposed under this background, the purpose is to research and development new AgSnO2 preparation technology, for the silver based electric contact materials to provide theoretical basis for the development and application.

\section{Silver based electric contact materials related technology}

Nearly 30 years, silver based contact materials research and development is an important content of developing alternative to traditional AgSnO2 contact materials of new materials [3]. The main reason has two: one is the requirement of environmental protection, AgSnO2 inevitably produced in the process of manufacture and use. Tin poison., has been increasingly the attention of people, some western developed countries have banned on the part of the household appliances and automotive electronics use silver oxide partial materials; Second, electrical use of performance requirements, while silver oxide $f$ materials have moderate load electrical universal contact, but in fusion welding resistance, arc resistance performance aspects such as the loss also exposed more and more difficult to meet the electric switch of contact materials of miniaturization, high reliability, long life and other harsh electrical performance requirements. Silver based electric contact methods mainly 
include alloy oxidation, powder metallurgy method, coprecipitation method, four kinds of powder alloy oxidation method.

Gold in the oxidation process. Oxidation in the alloy is a kind of more advanced manufacturing process method of electric contact material, is widely used both at home and abroad. In AgMeO contact material, silver cadmium oxide (AgCdO)) and silver zinc oxide (AgZnO) using this method, many with silver cadmium oxide contact materials (AgCdO) 's manufacturing process, for example [4], the basic process is shown in figure 1.

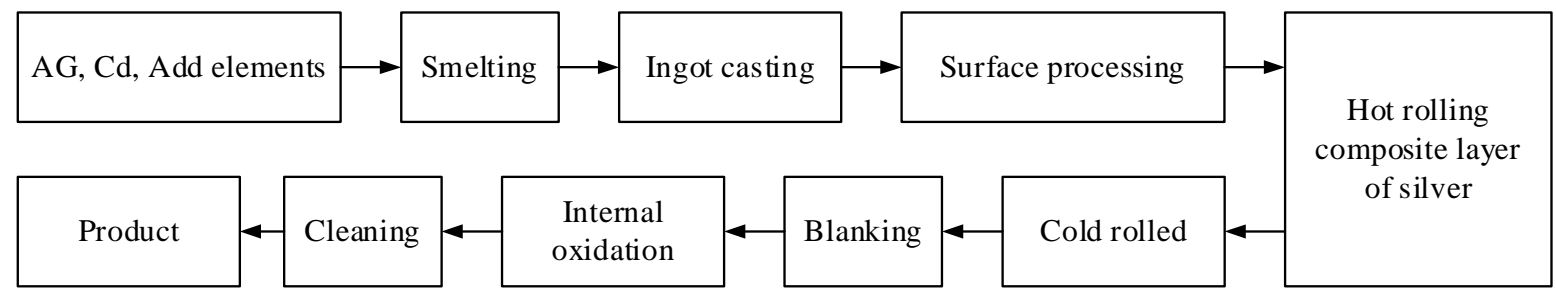

Figure 1. Gold in the oxidation process

The powder metallurgy method. AgMeO contact method of powder metallurgy materials especially for AgsnO2 contact materials is one of the most important methods. Common production method is to mix powder extrusion, pressing, sintering, to take advantage of the strip, in the powder metallurgy method, extrusion powder sintering process is more and more attention, has become the main trend of manufacturing technology [5]. Its process is shown in figure 2.

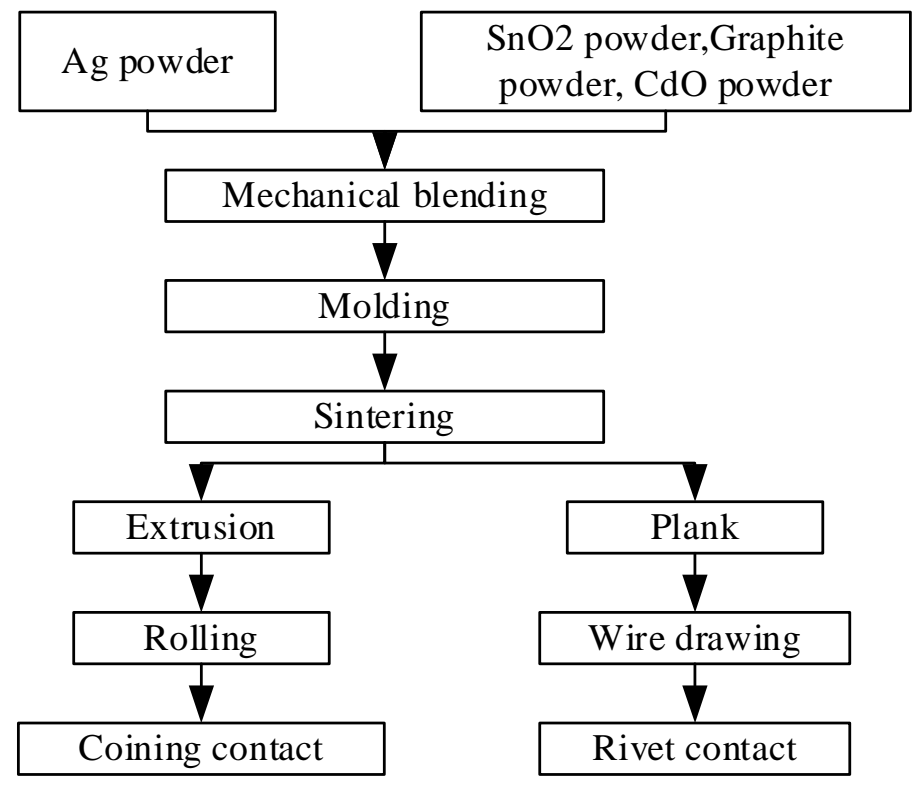

Figure 2. The powder metallurgy method

\section{The experiment design and analysis}

AgSnO2 electrical contact materials with particle size $<74$ um, purity $>99.5 \%$ of silver powder and other reagents as raw materials, preparation of content is respectively $5 \%, 8 \%$ and $10 \%$ of $\mathrm{SnO} 2 \mathrm{AgSnO} 2$ electricity contact materials. Powder metallurgy of AgSnO2 prepared by the electric contact material: using particle size $<74 \mathrm{um}$, purity $>99.5 \%$ of silver powder, granular $<50$ um $\mathrm{SnO} 2$ as raw materials, preparation of content is $5 \%, 8 \%$ and $10 \%$ of $\mathrm{SnO} 2 \mathrm{AgSnO} 2$ electricity contact materials. The raw material after mixer fully mixed, use steel mould for compact, after sintering, after pressure after burning, such as technology, cutting into the conductivity of the test sample is $6 \mathrm{~mm}$ thickness. After sintering, after pressure after burn of ingot hot extruded into a pole, cold drawing again into wire rod for microstructure analysis. Reaction synthesis of $\mathrm{SnO} 2$ particles 
is completed in the sintering process. By optical microscope, electron probe and transmission electron microscopy (sem) to block sample and microstructure analysis of wire rod sample, as shown in figure 3.

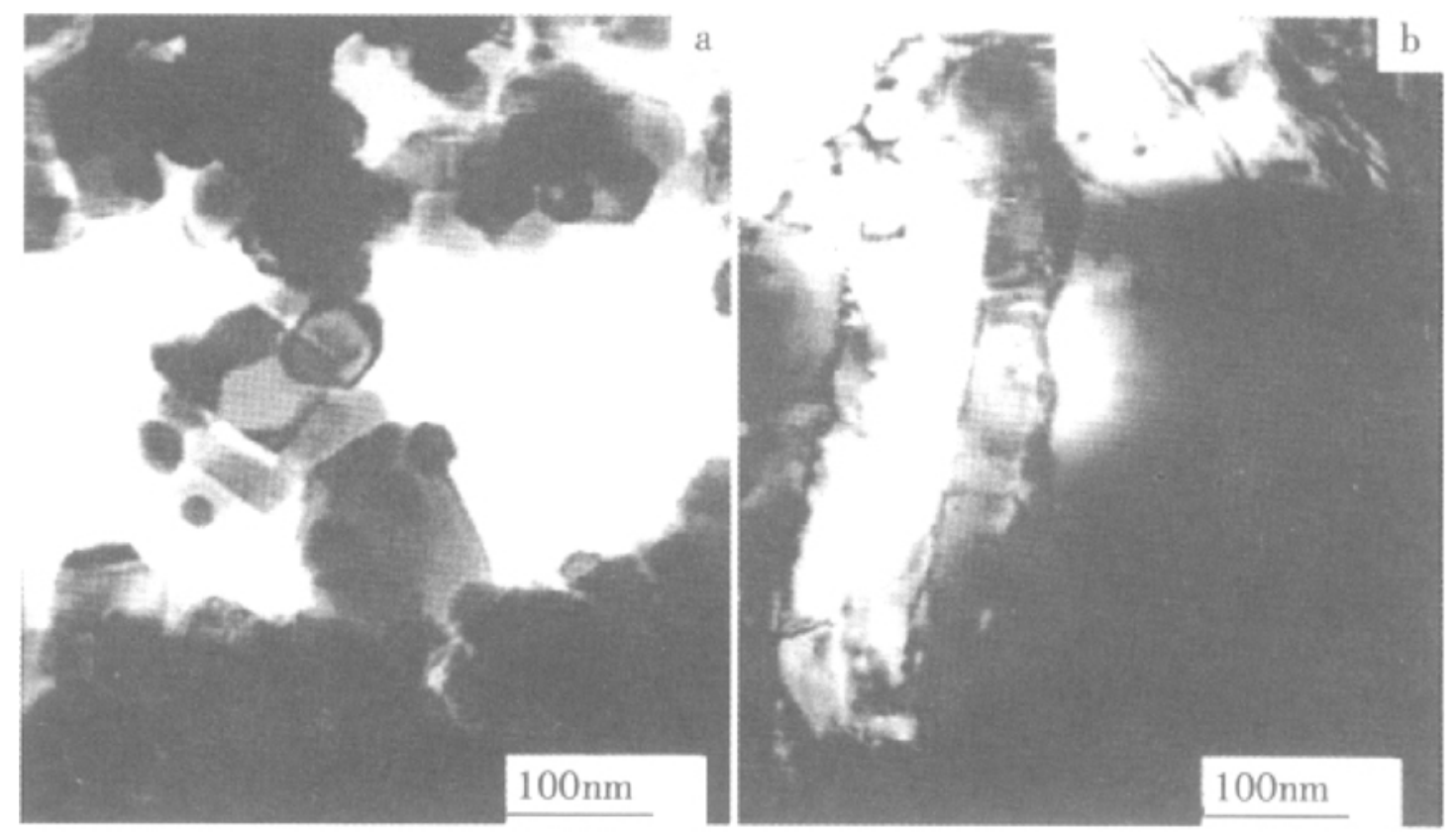

Figure 3. TEM image for AgSnO2

(a) Reactive synthesis (b) Power metallurgy

Figure (a) is synthesized AgSnO2 tem analysis results. From the point of electron microscope photos, $\mathrm{SnO} 2$ particles is very small. Electron diffraction pattern calibration these particles are tetragonal SnO2, attached to the small particles of cone spots are Ag, suggests that a small amount of $\mathrm{Ag}$ has to immerse himself in $\mathrm{SnO} 2$ particles at this time. According to the theory of metal plastic forming, small grains, organization between different grain, grain deformation is uniform, between different regions of deformation coordination and easily add fine grain structure of flow stress is higher, better toughness, this structure should be especially AgSnO2 electrical contact materials of a breakthrough. Figure (b) is powder metallurgy AgSnO2 material transmission electron microscopy (sem) analysis results. You can see from figure has a gross grain, calibration for Ag by electron diffraction of single crystal diffraction spots. Which can be concluded that in figure (b) the strip material is the grain Ag. This is due to the sample made by powder metallurgy method, Ag with $\mathrm{SnO} 2$ interface is bad, and transmission electron microscope samples need after grinding, double injection thinning, $\mathrm{SnO} 2$ particles fall off in this step, leaving only the matrix element of Ag.

\section{Conclusion}

Reaction synthesis of the samples prepared net-like, particle size distribution is uniform, little reunion phenomenon, the electrical conductivity of the material is good. Powder metallurgy of material particles prepared by the reunion phenomenon is serious, interface bonding is not good, so the electrical conductivity of the material are not nearly as reaction by synthetic materials. In addition reaction synthesis of materials prepared by due to the better interfacial wettability, interfacial combine closely, make the material has good corrosion resistance. Adopted in the process of preparation of materials in the processing of synthesis reaction with a relatively low initial strength and hardness, but with the increase of deformation rate, intensity changes faster, the initial processing is relatively easy. Hardness and elongation in deformation rate of $6 \%$ to $26 \%$ when appear abnormal phenomenon, the deformation rate was $16 \%$, hardness and elongation have extreme value, the processing performance is good, easy to pull material. 


\section{Reference}

[1] Haitao, Wang Jingqin1 Zhu Yancai1 Wang, and Zhao Jun. "Impact of Additives Bi on AgSnO_2 Contact Material [J]." Transactions of China Electrotechnical Society 1 (2011): 007.

[2] Liu, X. M., et al. "Characteristics of nano Ti-doped SnO2 powders prepared by sol-gel method." Materials Science and Engineering: A426.1 (2006): 274-277.

[3] Monnier, A., et al. "A coupled-field simulation of an electrical contact during resistance welding." Electrical contacts-2006, proceedings of the fifty-second ieee holm conference on. IEEE, 2006.

[4] Boettcher, Robert Donald. Arcing Failure of ROHS Compliant Electromagnetic Relays. Diss. 2012.

[5] Borkowski, P., and E. Walczuk. "Computerized measurement stands for testing static and dynamic electrical contact welding." Measurement 44.9 (2011): 1618-1627. 